\title{
Sleeve-Gastrektomie - Technik, Sicherheit, Erfolg, Trends
}

\author{
Peter Djalali Dieter Birk \\ Chirurgische Klinik, Evangelisches Krankenhaus Zweibrücken, Deutschland
}

\section{Schlüsselwörter}

Laparaskopische Sleeve-Gastrektomie · LSG · Adipositas · Sicherheit · Operationstechnik · Indikation

\section{Zusammenfassung}

Obwohl über die laparoskopische Sleeve-Gastrektomie (LSG) noch keine ausreichenden Langzeitergebnisse vorliegen, wird diese Operation mit zunehmender Euphorie durchgeführt. Multicenter-Studien sind in der Rekrutierungsphase, Langzeitergebnisse werden erst in 2-3 Jahren erwartet. Die LSG wird allgemein als eine zügig durchführbare und wenig traumatisierende Operation angesehen. Es gelten aber die gleichen Grundsätze der laparoskopischen Chirurgie: sorgfältige Präparationen, Vermeidung von kreuzenden Klammernahtreihen und das frühe Erkennen und Beherrschen von Leckagen. Nur so kann ein hoher operativer Standard gewährleistet werden. Wir verwenden einen 32-Charrière-Magenbougie; daraus sollte ein circa 80-100 ml großer Schlauchmagen und ein Magenresektat mit mindestens $1000 \mathrm{ml}$ Füllungsvolumen resultieren. Werden diese Benchmarks angestrebt, können Übergewichtsverluste (EWL) von circa $60 \%$ nach 1 Jahr erzielt werden.

\section{Einleitung}

Die laparoskopische Sleeve-Gastrektomie (LSG) hat als ein neues und eigenständiges Verfahren der bariatrischen Chirurgie in den Adipositas-Zentren Einzug gehalten. Hiermit konnte dem Anspruch des Patienten auf eine weitere restriktive Methode ohne die Verwendung eines Implantats Rechnung getragen werden. Entstanden aus der Magenstrasse-Mill-Prozedur, die besonders in Großbritannien in den 1970er und 1980er Jahren Anwendung fand, etablierte sich die LSG zu Beginn des Millenniums als selbständi-

\section{Key Words}

Laparoscopic sleeve resection · LSG · Obesity · Safety · Operative technique $\cdot$ Indication

\section{Summary}

Sleeve Gastrectomy - Technique, Safety, Success, Trends Despite the lack of sufficient data concerning the long-time results, the morbidity, and the mortality of laparoscopic sleeve resection, this operation is performed with growing enthusiasm. However, general principles of laparoscopic surgery need to be observed to ensure good results. This article describes an operative technique and indication for the operation. Attention must be turned on the complete liberation of the dorsal aspect of the stomach, the absence of crossing staple lines, and the competent handling of intraoperative leakages. The use of a $32 \mathrm{Fr}$ bougie results in a residual stomach volume of about $80-100 \mathrm{ml}$. Due to a good operative technique and a competent follow-up, excess weight loss (EWL) of about $60 \%$ can be achieved within the first postoperative year.

ger Eingriff. Es wurde eine deutliche und ausreichende Gewichtsabnahme bei Patienten beobachtet, die eigentlich eine biliopankreatische Diversion (BPD) mit duodenal Switch (DS) erhalten sollten. Das Unterlassen der malabsorptiven Komponente als Zweiteingriff resultierte hieraus [1]. Zum einen besticht diese Art der Restriktion durch einen raschen Excess Weight Loss (EWL) von circa 49\% nach 6 Monaten und circa 60\% EWL nach 1 Jahr [1], zum anderen wird die vermeintlich einfache Durchführung und Erlernbarkeit der Operation als Vorteil gesehen. Schon nach wenigen Assistenzen kann der geübte Laparoskopiker die Kombi-

\section{KARGER \\ (C) 2009 S. Karger GmbH, Freiburg \\ Fax +497614520714 \\ E-mail Information@Karger.de www.karger.com/ofa

PD Dr. Dieter Birk

Chirurgische Klinik, Evangelisches Krankenhaus Zweibrücken Obere Himmelsbergstraße 38, 66482 Zweibrücken, Germany

Tel. +49 6332 42-21 20, Fax -2134

D.Birk@evkhzw.de 
nation von Teilabschnitten, wie sie auch bei Gastric Banding und Fundoplikatio zum Zuge kommen, anwenden. Aus der Qualitätssicherungsstudie der Deutschen Gesellschaft für Chirurgie der Adipositas e. V. geht hervor, dass im Jahr 2007 durchschnittlich circa 93 min für diese Operation erforderlich waren, und diese damit im Schnitt 15 min länger dauerte als die Implantation eines anpassbaren Magenbandes (Laparoscopic Adjustable Gastric Banding; LAGB) [2, 3]. Die Materialkosten von circa 1000,- EUR sind nicht unerheblich, jedoch mit den Kosten eines LAGB vergleichbar.

Komplikationen, wie z. B. Anastomoseninsuffizienz, Ulzera, innere Hernien, sind nicht mit der LSG assoziiert. Durch den Einfluss auf den Ghrelinspiegel ergibt sich ein hormoneller Vorteil gegenüber der LAGB [4]. Die Sterblichkeitsrate ist mit 0-4\% angegeben und Hauptkomplikationen, wie Klammernahtinsuffizienz, Randblutung oder postoperativer gastroösophagealer Reflux, entstehen bei 0-36\% der Operierten [5].

Sind dies also Gründe für die große Euphorie? Ergebnisse aus prospektiven Multicenter-Studien werden dringend benötigt. Positive Trends ergeben sich bezüglich Effizienz, Wirkmechanismus und Indikation. Anders als bei den bekannten Verfahren besteht bereits eine große Euphorie, ohne valide Kenntnis über Langzeitergebnisse und Komplikationsraten zu haben. Welcher Adipöse profitiert also von der LSG? Welche Technik ist die Sicherste?

\section{Indikation}

Die LSG führt zur Restriktion und zur initialen Senkung des Serum-Ghrelinspiegels [4]. Sie limitiert den Vielesser mit BMI $>40 \mathrm{~kg} / \mathrm{m}^{2}$, aber auch der Patient mit BMI $>35 \mathrm{~kg} / \mathrm{m}^{2}$ und komorbidem Diabetes, Schlafapnoe oder Hypertonie kann hiervon profitieren [1]. Superobese Patienten $\left(\mathrm{BMI}>50 \mathrm{~kg} / \mathrm{m}^{2}\right)$ erhalten durch Downstaging des BMI eine bessere Voraussetzung für BPD oder laparoskopischen Magenbypass (LGBP) [6, 7]. Bei Bandversagen und Beibehalten des restriktiven Ansatzes wird die LSG favorisiert. Jugendliche, Kinder sowie ältere Patienten sind nicht von der Indikation für eine LSG ausgeschlossen. Wahrscheinlich wird sich gerade die LSG für den jugendlichen Erwachsenen zum Eingriff der Wahl entwickeln. Kontraindikationen sind erosive Gastritis und Magenulkusleiden; auch bei der Refluxkrankheit mit großer Hiatushernie gilt eine strenge Indikationsstellung. Patienten mit Ösophagus- oder Magenvarizen, angeborenen oder erworbenen Teleangioektasien und gewebeassoziierter autoimmuner Antikörperkrankheit (Lupus, Sklerodermie) sind ungeeignet für die LSG. Der hauptsächlich Sweeteater profitiert von der LSG, wie von den anderen restriktiven Operationsverfahren, nur begrenzt.

\section{Angewandte Verfahren an der Chirurgischen Klinik des Evangelischen Krankenhauses Zweibrücken}

Die Operation wird unter Intubationsnarkose durchgeführt. Gelagert wird der Patient in der Beach-Chair-Position. Der Operateur steht zwischen den Beinen des Patienten. Das Pneumoperitoneum

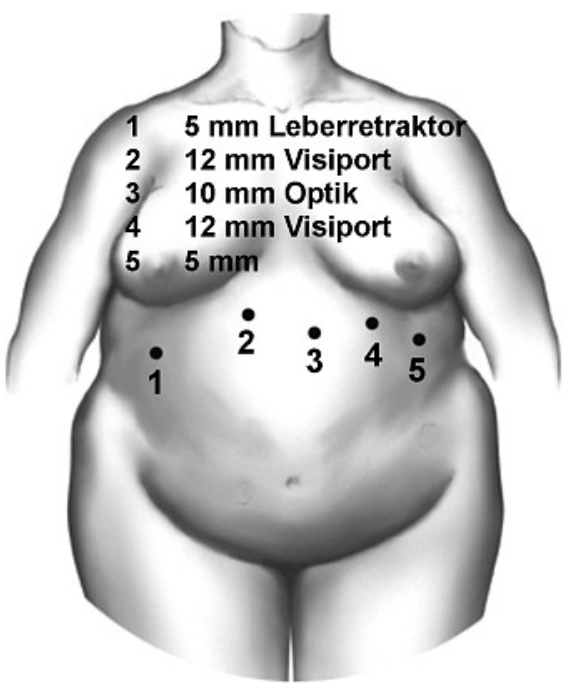

Abb. 1. 5-Trokar-Platzierung in der LSG.

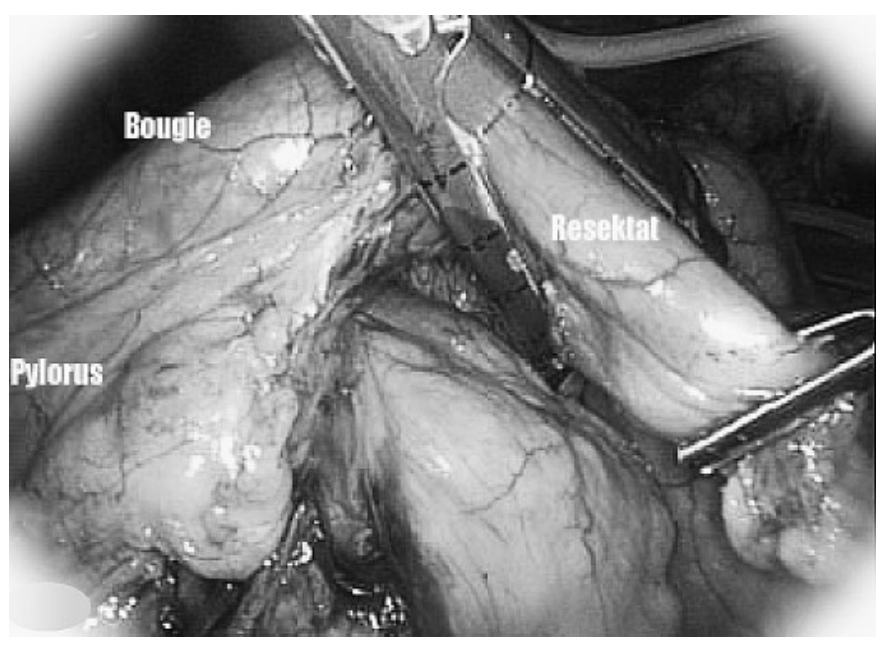

Abb. 2. Intraoperatives Bild.

entsteht durch Punktion mit der Veres-Nadel paraumbilikal, selten links subkostal. Die in Abbildung 1 dargestellte 5-Trokar-Platzierung hat sich im Regelfall bewährt. Über den äußeren subkostalen 5-mm-Trokar (1) hält der Retraktor die Leber zur Seite. Nach Vervollständigung des Trokar-Setups unter Sicht wird zunächst der Eingang in die kleine Bursa durch Dissektion des gastrokolischen Ligaments ermöglicht. Dieser entsteht circa $8 \mathrm{~cm}$ proximal des Pylorus und wird mittels Ultrazision entlang der großen Kurvatur, jedoch erst nach Platzierung eines 32-Charrière-Bougies, bis zum His-Winkel erweitert. Hierbei ist die Adhäsiolyse an der dorsalen Magenwand zum Pankreasoberrand und zum Milzpol entscheidend, um bei den folgenden Operationsschritten Verletzungen dieser Organe auszuschließen. Die ersten beiden blauen Magazine werden über den Trokar (2) sehr knapp entlang des Kalibrationsschlauchs, circa $6 \mathrm{~cm}$ proximal des Pylorus geschossen (Abb.2). Weitere blaue Staplermagazine dissektieren den Magen sehr knapp entlang des Bougies (Abb. 3) bis zum linken Zwerchfellwinkel, sodass lediglich ein Schlauchmagenreservoir zurückbleibt. Insgesamt werden circa 6 Ladeeinheiten blaues Magazin (45-60 mm) 


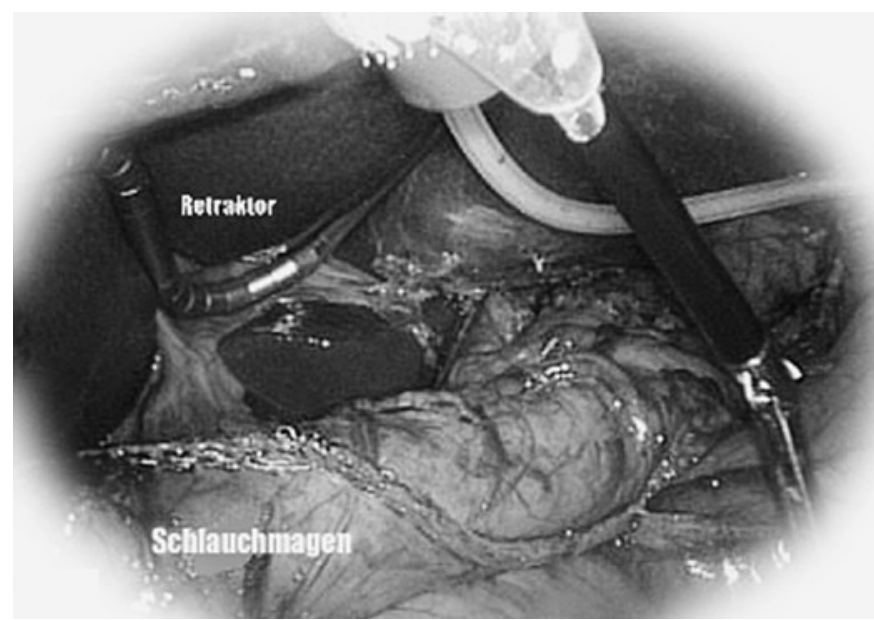

Abb. 3. Dissektion des Magens entlang des Bougies.

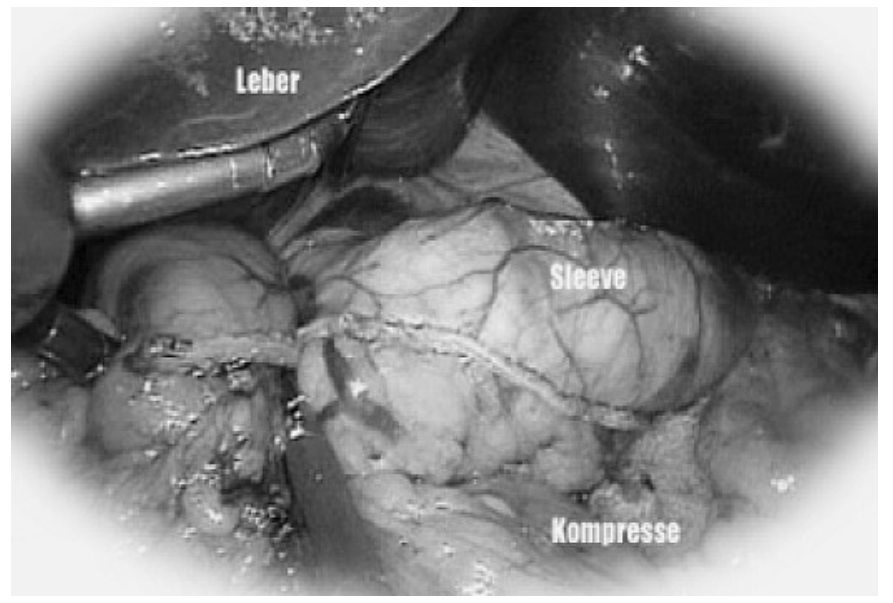

Abb. 4. Dichtigkeitskontrolle des Schlauchmagens.

verwendet. Abschließend wird das Magenresektat in einen Bergebeutel verbracht. Die Dichtigkeitskontrolle des Schlauchmagens erfolgt durch Befüllung von blauer Lösung durch den Anästhesisten (Abb. 4). Mögliche Leckagen werden in Einzelknopftechnik übernäht. Abschließend wird der Oberbauch ausgespült und eine 6er-Easy-Flow-Drainage eingelegt. Das Entfernen der Trokare unter Sicht geschieht simultan mit der Extraktion des Magenresektats durch eine kleine erweiterte Portinzision. Nach Verschluss der Trokarpforten wird das Resektat gefüllt und das Volumen dokumentiert.

\section{Erfahrungen und Probleme in der Chirurgischen Klinik des Evangelischen Krankenhauses Zweibrücken}

Der vom Anästhesisten transoral eingebrachte Bougie gibt die Richtung und das Ausmaß der Dissektion vor. Um dessen Transsektion zu vermeiden, ist vor jedem Auslösen des Staplers eine Sondenfehllage auszuschließen. Das Auslösen des Staplers sollte langsam und gleichmäßig erfolgen, um einen sicheren Gewebeverschluss zu erreichen. Ein Kreuzen der Staplerlinien ist zu vermeiden. Im Fundusbereich wird die Gewebemanschette etwas größer belassen. Bei einer durch Blautest gesicherten Undichtigkeit dieser kritischen Stelle kann das dadurch erhaltene Gewebe die Übernähung vereinfachen. So erübrigt sich für uns die Verwendung von Nahtverstärkungen wie Seamguard ${ }^{\circledR}$ (WL Gore, Flagstaff, AZ, USA). Ist eine arterielle Blutung aus der Nahtreihe zu beobachten, gilt es, diese bei Persistieren zu übernähen. Der EWL-Erfolg korreliert mit einer ausreichenden Magenresektion $(\geq 1000 \mathrm{ml}$ bei Männern und $\geq 800 \mathrm{ml}$ bei Frauen) bzw. einem Magenschlauch von maximal 80-100 ml Volumen.

Das geschickte Setup der Trokare spart Operationszeit und determiniert zudem den Komfort des Operateurs. Nach Magenballonbehandlung (BioEnterics Intragastric Balloon, BIB) sollte wegen der Dilatation und des Wandödems des Magens zunächst mindestens 4 Wochen abgewartet werden. Bei der simultanen Bandentfernung wegen Bandversagens müssen speziell eine mögliche Wandhypertrophie sowie Wanderosionen in der Manschettenrinne beachtet werden. Die Dissektion an der deutlich verdickten Magenwand sollte dann mit einem grünen Staplermagazin durchgeführt werden.

\section{Schlussfolgerung}

Die laparoskopische Schlauchmagenbildung scheint ein sicheres und effektives Verfahren in der bariatrischen Chirurgie zu sein. Kenntnisse über Langzeiteffekte der Gewichtsabnahme, dessen Ausmaß und Dauer werden nur durch ständige Erhebungen und Aktualisierung von Daten, die in Qualitätssicherungsstudien einfließen, gewonnen. Das Adipositaszentrum der Chirurgischen Klinik des Evangelischen Krankenhauses Zweibrücken behandelt bei gegebener Indikation adipöse Patienten durch LSG mit der oben dargestellten Methode und Technik, die sich dort bewährt haben. Erfolg und Misserfolg der Sleeve-Gastrektomie hängen jedoch nicht nur von einer subtilen Operationstechnik ab, sondern sind maßgeblich an eine exakte und lebenslange Nachsorge der Patienten gebunden, an der Experten unterschiedlicher Disziplinen beteiligt sind.

\section{Disclosure}

Die Autoren geben keine Interessenkonflikte an. 


\section{Literatur}

1 Nocca D, Krawczykowsky D, Bomans B, Noel P, Picot MC, Gagner M, et al: A prospective multicenter study of 163 sleeve gastrectomies: results at 1 and 2 years. Obes Surg 2008;18:560-565.

2 Stroh C, Birk D, Flade-Kuthe R, Frenken M, Herbig B, Höhne S, Köhler H, Ludwig K, Pick P, Horbach T, Krause S, Schäfer L, Weiner R, Wolff S, Wolf AM, Schmidt U, Manger T: Quality assurance in bariatric surgery in Germany - results of the German multicentre trial 2005 and 2006. Zentralbl Chir 2008;133(5):473-478.
3 Stroh C, Manger T: Auswertungsstudie der Qualitätssicherungsstudie «Operative Therapie der Adipositas» Jahrgang 2007:42.

-4 Langer FB, Reza Hoda MA, Bodjalian A, Felberbauer FX, Zacherl J, Wenzl E, Schindler K, Luger A, Ludvik B, Prager G: Sleeve gastrectomy and gastric banding: effects on plasma Ghrelin levels. Obes Surg 2005;15:1002410029.

5 Deitel M, Crosby RD, Gagner M: The first International Consensus Summit for Sleeve Gastrectomy (SG), New York City, October 25-27, 2007. Obes Surg 2008;18:487-496.
6 Almogy G, Crookes PF, Anthone G: Longitudinal gastrectomy as a treatment for the high-risk super-obese patient. Obes Surg 2002;12:492-497.

7 Milone L, Strong V, Gagner M: Laparoscopic sleeve gastrectomy is superior to endoscopic intragastric balloon as a first stage procedure for super-obese patients (BMI $\geq 50)$. Obes Surg 2005; 15:612-617. 